\title{
Trigon Panel Size Optimization Studies
}

\author{
A. Scott Howe, $\mathrm{PhD}^{*}$ \\ Plug-in Creations Architecture, LLC \\ Hong Kong University Dept. of Architecture \\ Ian Gibson, PhD. ${ }^{\dagger}$ \\ National University of Singapore Dept. of Mechanical Engineering
}

The optimal Trigon panel edge size will depend on a variety of factors, including mechanism sizes, proposed panel payloads, shielding, dust control, and human factors. The Trigon system allows for a kit-of-parts approach to habitat construction and vehicle design. Trigon panels may be fitted with mobility systems and used to construct habitats, vehicles, ISRU "Cassette factories", and other permanent structures. This paper discusses panel size optimization with respect to these potential uses, introduces a spreadsheet model for multivariate data input, and describes a series of studies that have been conducted to determine the effect of variable world reference frame acceleration scenarios (surface gravities) on panel edge dimensions.

\section{Introduction}

NASA Space architects have suggested a three-phase construction roadmap for planetary surface construction (Kennedy 2002). The first phase is Class I pre-integrated structures that are ready to use upon delivery. Class II kitof-parts structures will then be phased in, allowing for larger structures that require some assembly, and will become a transitional form of structures preparatory for Class III in-situ structures using native material. In the harsh environment of space, robotically assembled, or self-assembling structures will be necessary (Cohen \& Kennedy 1997).

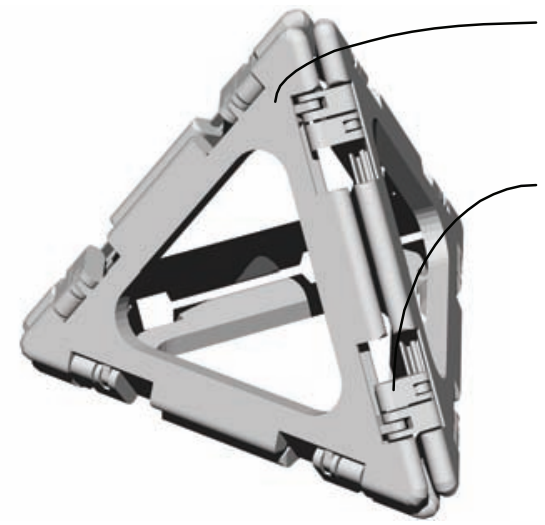

Panels with uniform edges (triangles, squares, etc)

Actuators at edges (connector arms) couple with adjacent panels

Panels stack flat for

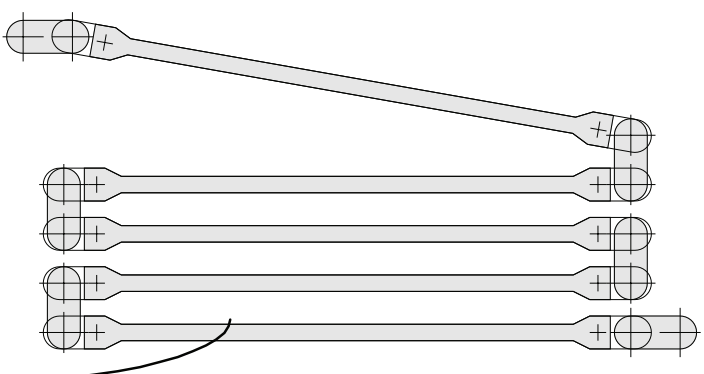
transporting

Figure 1: Trigon constructions unfold and stack for compact transport

The Transformable Robotic Infrastructure-Generating Object Network (Trigon) modular robotic construction system falls into the Class II category (Howe 2002), and consists of panel-based units that have edge actuators for linking to other panels. The edge actuators are hinged with an offset axis in such a way that any two panels can be orientated from 0 to 360 degrees in relation to each other. This ability allows the panels to be stacked during transportation, to keep the payload small and compact. Figure 1 shows a simple volume constructed of Trigon triangular panels, and illustrates how the panels can be folded and stacked.

\footnotetext{
* Assistant Professor, HKU Department of Architecture, ash@plugin-creations.com, AIAA member

${ }^{\dagger}$ Associate Professor, NUS Department of Mechanical Engineering, mpegi@nus.edu.sg
} 
The edge manipulator actuators control the panel with revolute motion in relation with neighboring panels. These manipulators are called connector arms. Two panels will mate with each other by nesting connector arms, with one panel taking hierarchical dominance over the other by moving its arms to the outside, and the other panel moving the arms to the inside, so that both sets of arms mutually clasp each other. The revolute actuator in each arm set can then cause the panels to reconfigure their orientation with respect to each other. Using this technique, Trigon panels can be added to the structure at any location and use the motion of the connector arms to latch on to already completed portions of the structure, and travel or "tumble" end over end, avoiding other traveling panels, to find its own specified location (Figure 2).

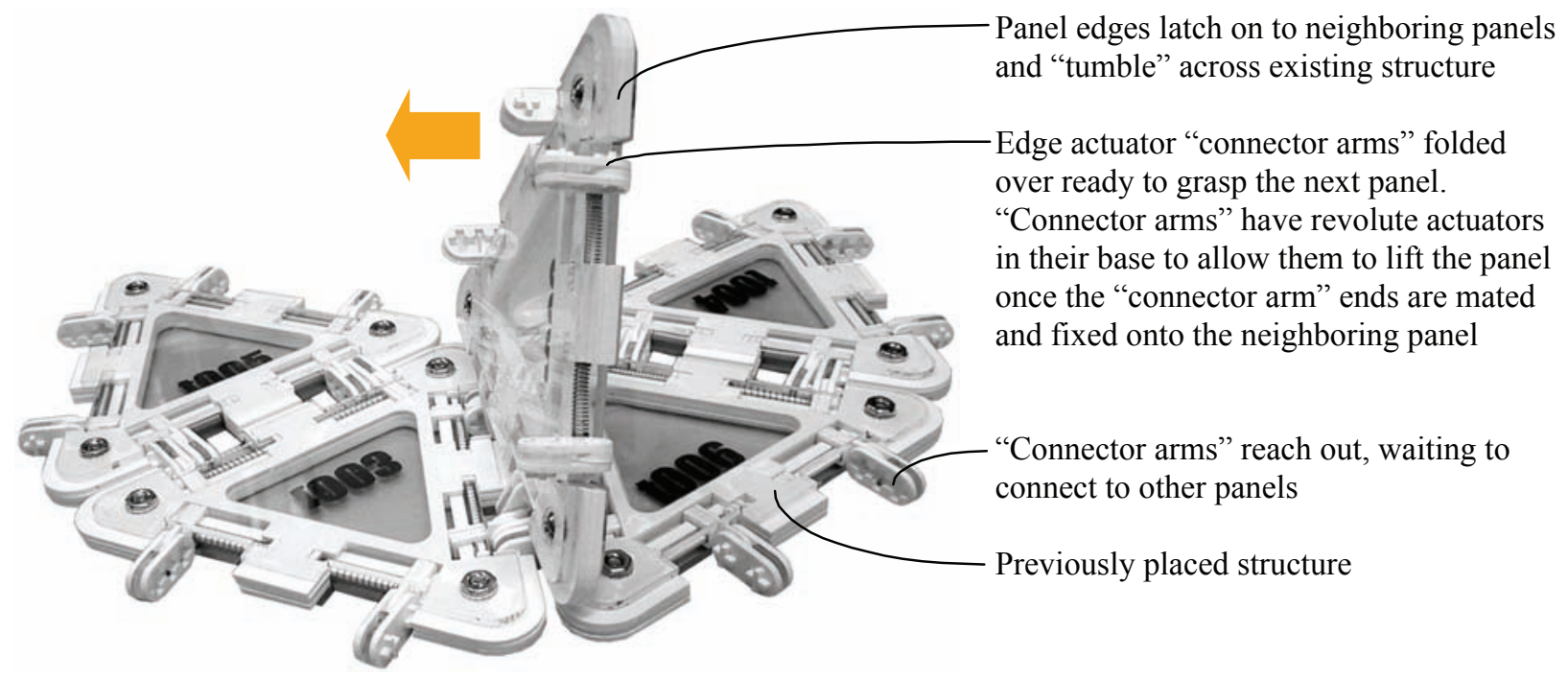

Figure 2: Trigon panels self-assemble by "tumbling" over already completed portions of the structure

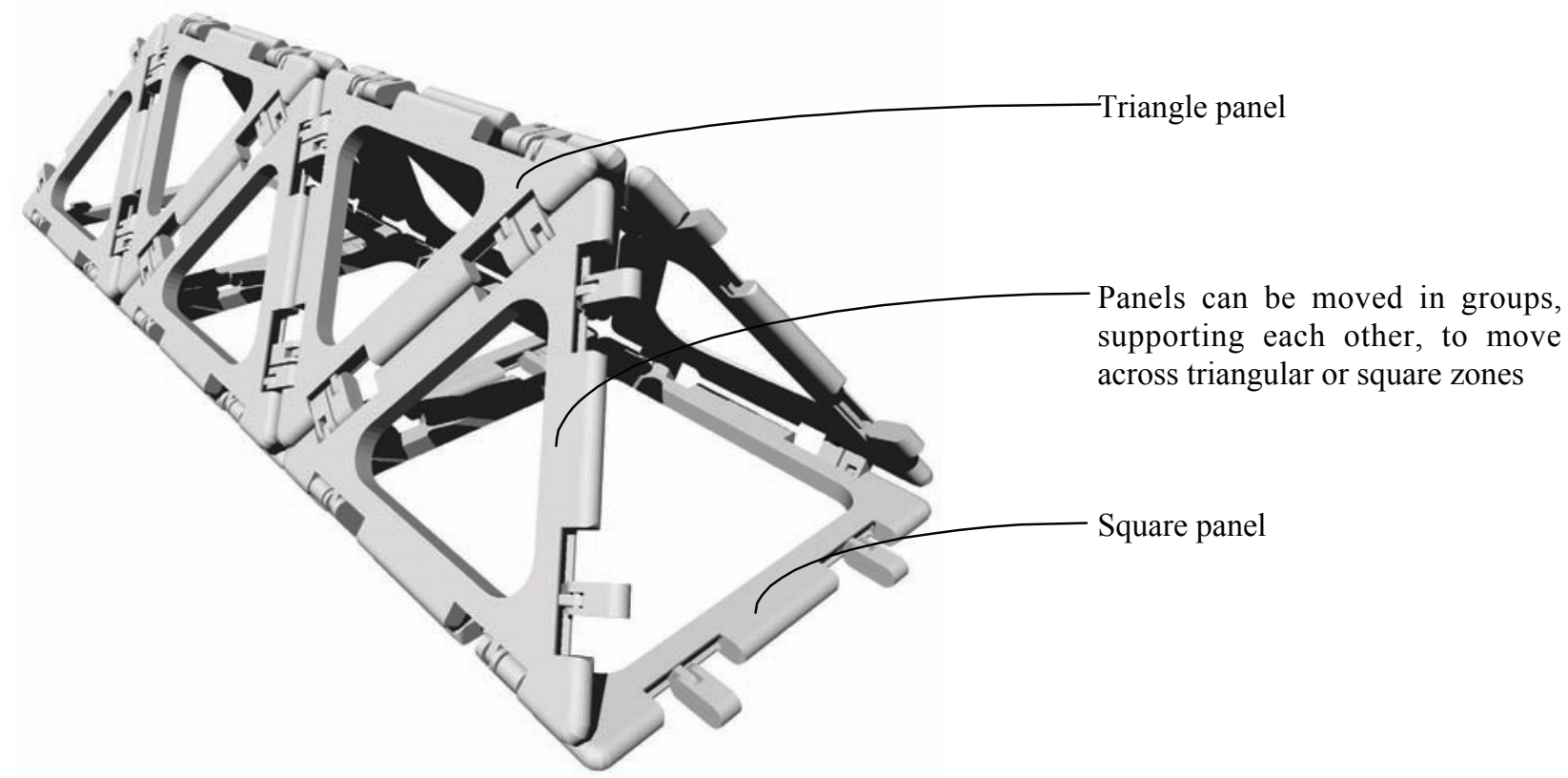

Figure 3: Trigon panels can be used to assemble a large variety of stable geometries, including trusses, vaults, domes, cylinders, etc.

Using only square and equilateral triangular panels, self-assembling domes, cylinders, trusses, and many other stable construction geometries can be autonomously assembled (Figure 3). These structures can function as 
reconfigurable secondary structure (Yip \& Howe 2003) or in combination with an inflatable pressure barrier lining (Figure 4) can be used as primary pressure vessel structures (Lai \& Howe 2003).

The Trigon panels can piggyback additional infill elements, that can be stacked with the panels, and moved about and relocated using the mobility of the connector arm mechanisms. These infill elements are called payload panels, and can include fold-out wheel and suspension systems, antenna, shielding, tankage, equipment, etc. to produce full mobility and fitout for Trigon structures. Payload panels can also contain packaged processes for "cassette factories" that may be used in ISRU processes (Howe 2005).

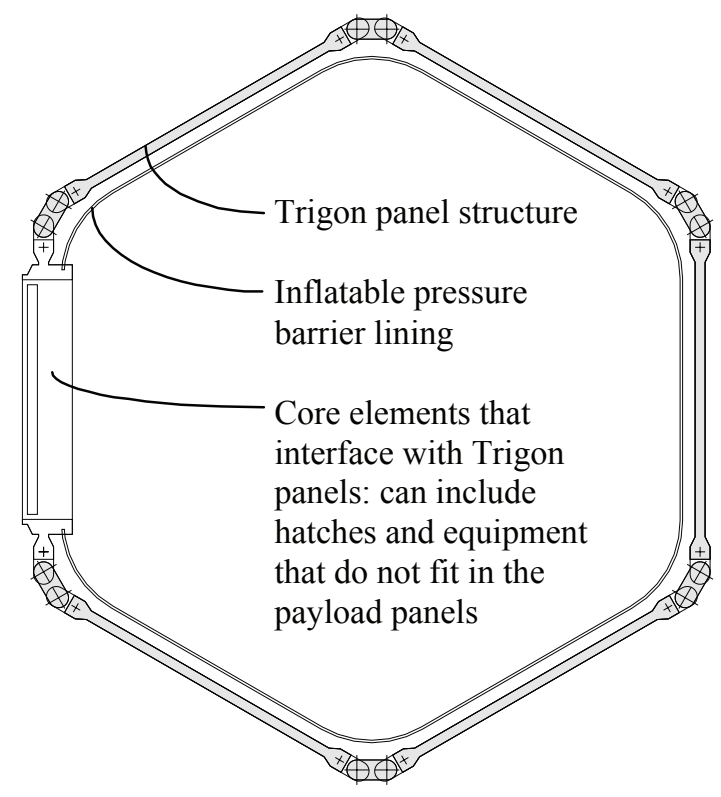

Figure 4: Trigon shell with inflatable lining

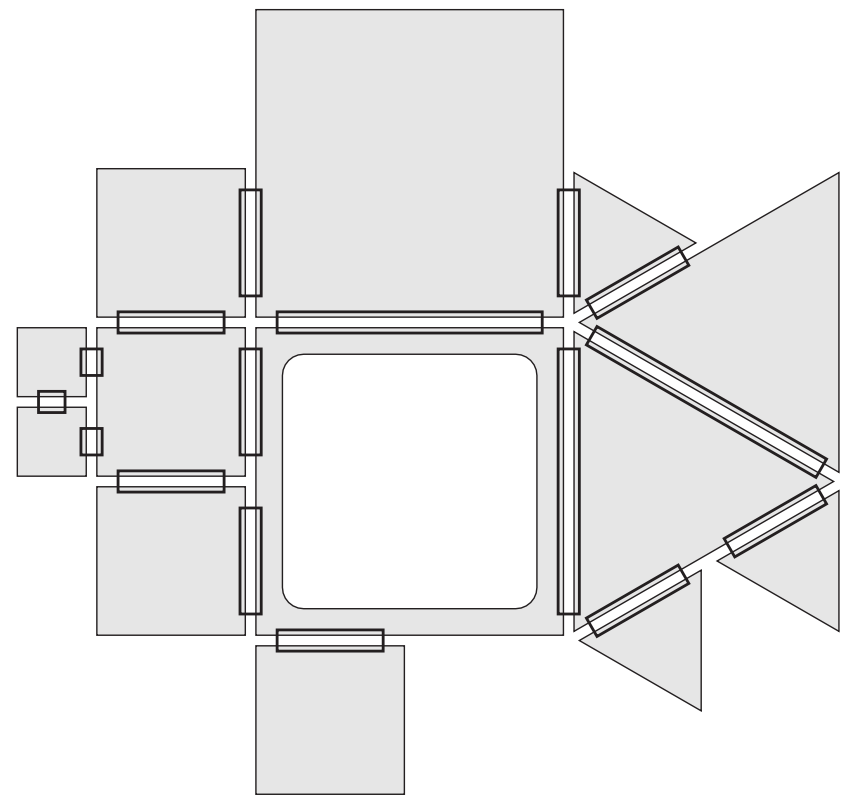

Figure 5: Trigon panel optimum size?

\section{Panel Sizes}

Trigon triangular and square panels, and the payload panels that fit them, may be used to construct habitats, frames, rovers, landing systems, equipment enclosures, construction equipment, and a variety of other uses (Howe, Gibson 2006a). The development of the panel systems will require multiple studies on the optimum size that the panels should be constructed. The size of the panels may be constrained by torque and gearing systems in the connector arms, payload equipment size requirements, mass loads, and human ergonomics. Conceptually, the Trigon system may be able to accommodate multiple sizes in a single structure, that halve at each edge in a fractal organization (Figure 5). However, a range of parametric constraints applied to panels of uniform size can provide a means of rigorous comparison by incrementally stepping up the values in each set. In this investigation, a numerical model of the Trigon panel was developed that can generate values for specific sizes, or tabulated into incremental values in a spreadsheet.

Figure 6 shows the geometry parameters for the triangle panels, including kinematic elements (Howe, Gibson 2006b), dependent variables, and parameter definitions. Some aspects of the diagram are exaggerated for clarity. The geometry parameters for the square panels are shown in Figure 7.

\section{A. Constraints}

Many of the parameters are dependent on a few variables, such that sizes can be adjusted according to mass, payload, torque, and ergonomic constraints. It must be stressed that most of the actual constraints are unknown at this time, therefore the following are suggested as possible future considerations. The analysis and panel size optimization study discussed in this paper allows for these future unknowns.

\section{Payload Constraints}

The payload panel mounts into the triangular or square opening in the center of the Trigon modular robotic panel. The Trigon panel supplies power to the payload panel, such that a variety of electrically-powered actuators, implements, manipulators, instruments, wheels, mobility systems, communication equipment, photovoltaic cells, 
batteries, tankage, collectors, encapsulated processes, shielding, and other functions can be inserted into system with modular flexibility. The sizes of these various functions are unknown at this time, but could influence overall panel sizing when such information becomes available.

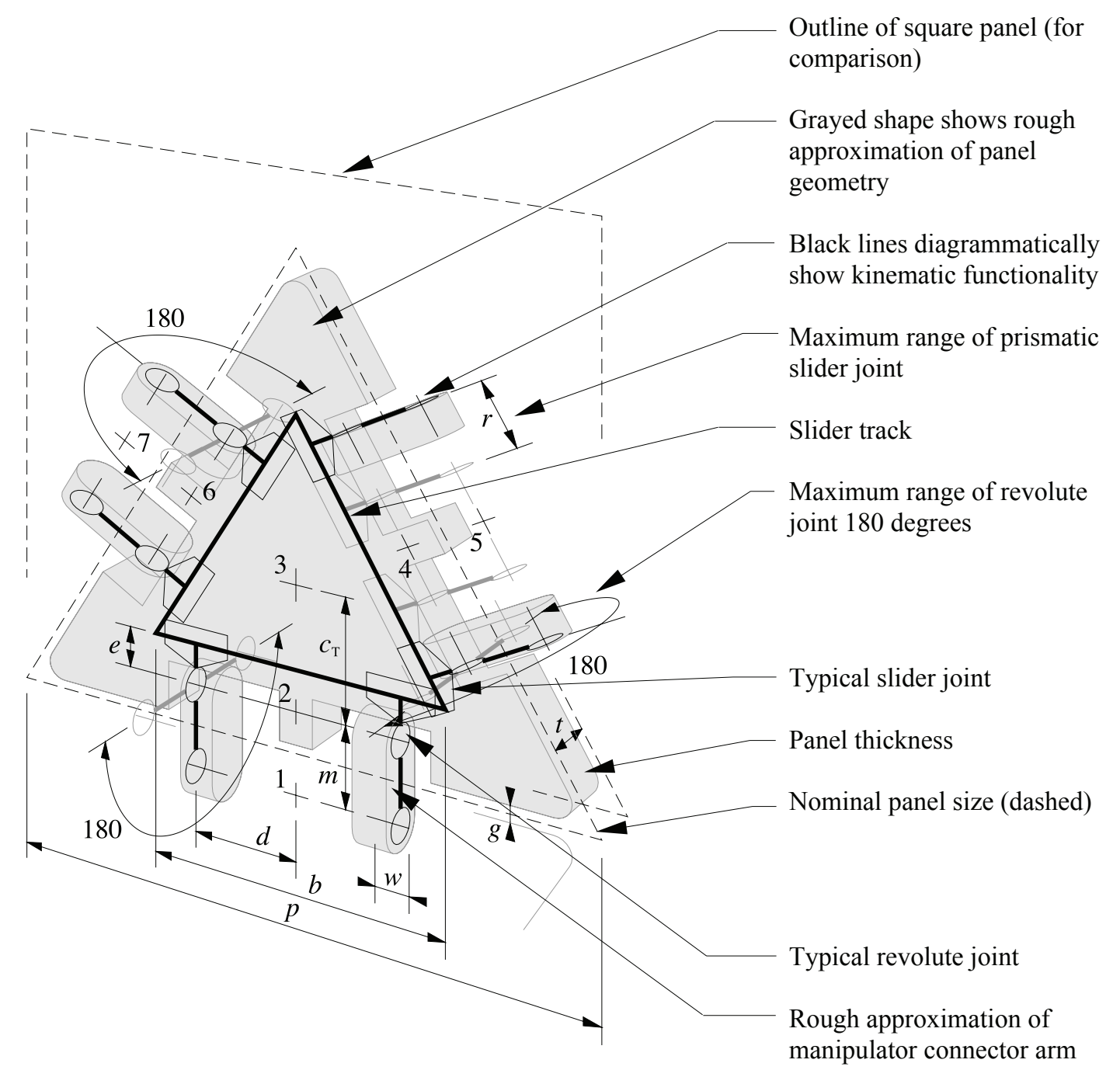

$t=$ panel thickness

$g=$ gap between panels

$e=$ mechanism depth

$w=$ connector manipulator arm width

$m=t+g=$ connector arm manipulator link

Point 3 is the centroid. Points 1-

$b=7 w+t=$ payload edge length / effective slider length (minimum)

7 coincide with equivalent

$c_{T}=e+b / 2(\tan 30)=$ distance to centroid of traingle kinematic joint locations.

$d=b / 2=$ maximum distance of arm from center

$r=$ slider range $(0$ to $3.5 w)$

$p=b+2\left(\frac{e+(t+g) / 2}{\tan 30}\right)=$ nominal panel edge

Figure 6: Triangle panel geometry parameters 


\section{Mass Constraints}

The mass budget for each of the Trigon panels and payload panels could influence the overall panel sizing. This initial study assumes the panel mass to be uniform where the center of mass is at the geometrical center of the panel.

\section{Torque Constraints}

Sizes of motors, revolute actuators, sensors, and gearing mechanisms for the connector arms will be determined by panel mass values and planetary gravity acceleration. Practical ranges of motors and mechanism will ultimately affect the overall panel sizing. Also, the maximum torque is assumed to be required for a panel climbing upward; other studies may be required to analyze torque required for resisting downward movement.

\section{Ergonomic Constraints}

The Trigon panels can include hatches, windows, or other features that are dependent on ergonomic standards and function. For example, if the panel were to be sized according to the ISS standard hatch size, the square opening in the middle of the panel would need to be large enough to mount the standard hatch hardware. If, on the other hand, the ergonomic constraints of hatch sizes are not critical, the panels could be smaller or larger in size. These human-related ergonomic standards will influence the overall panel sizing.

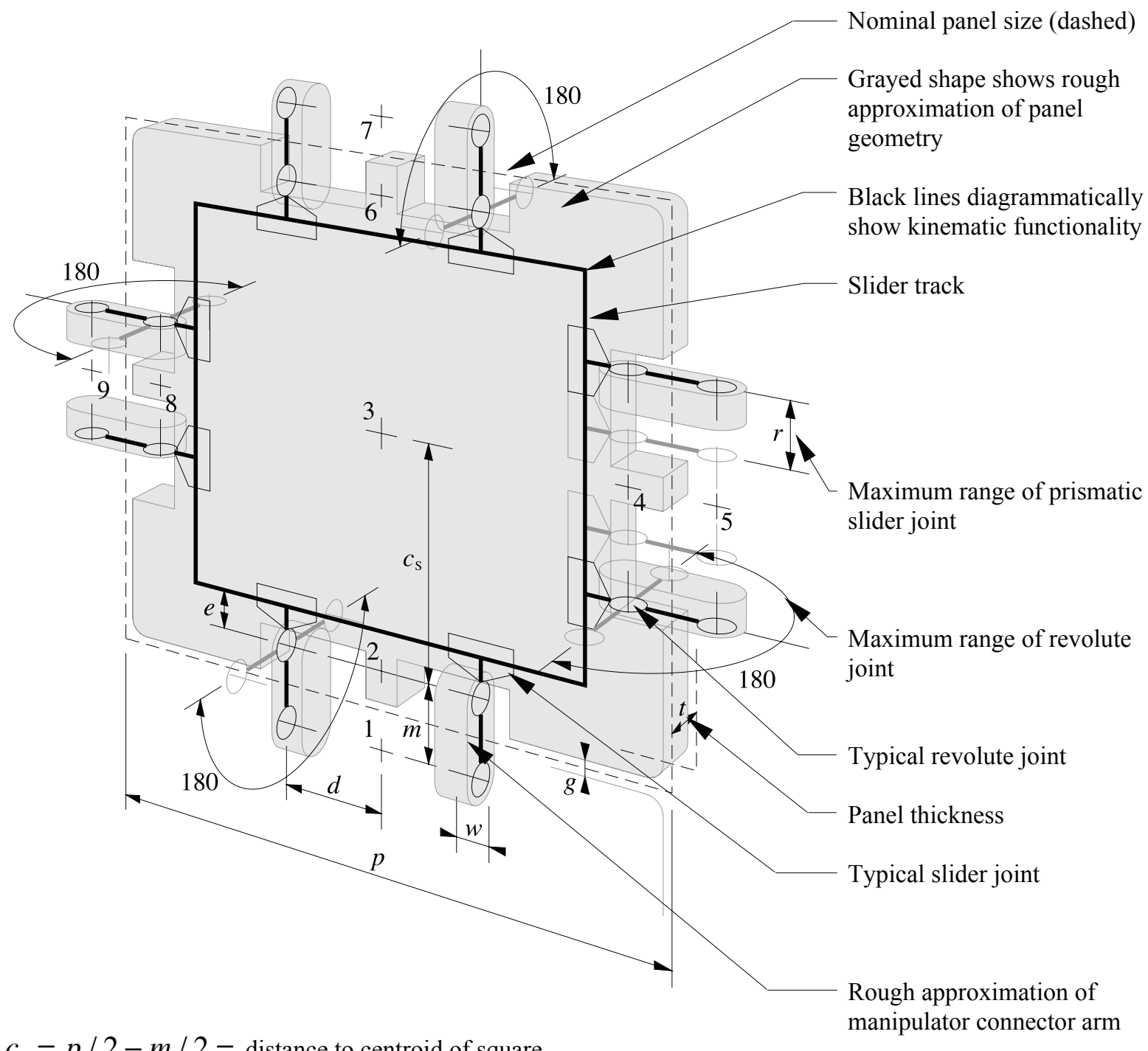

$c_{S}=p / 2-m / 2=$ distance to centroid of square

manipulator connector arm

\section{Figure 7: Square panel geometry parameters}




\section{A Mathematical Model for Optimum Panel Size Investigation}

Four base parameters were established: panel thickness $t$, gap between panels $g$, mechanism depth $e$, and connector arm width $w$. All other panel dimensions are derived or dependent upon these four parameters. Using the equations in Figure 6 and Figure 7, a mathematical model was developed for inputting the four base parameters, plus density and gravity acceleration values, whereby the rest of the dependent variables are generated. The model can be used to calculate values of a single set of inputs, or set to loop in an iterative generation of multiple solutions, each time incrementing one or more of the input values.

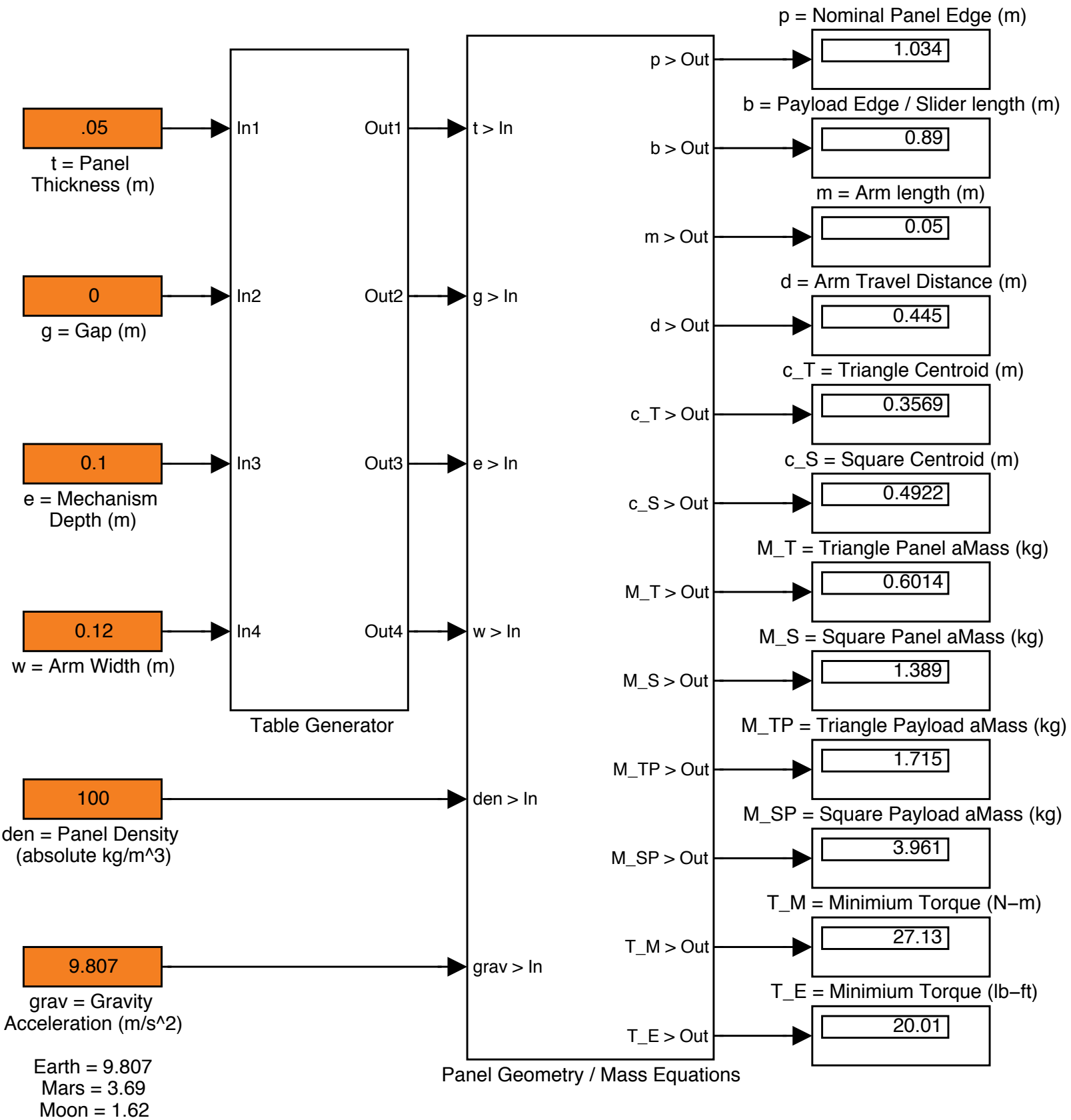

Figure 8: Panel size parameter control input (orange boxes) and output

The control panel for the model is shown in Figure 8. The program is used by inputting values in the orange boxes (on the left side of Figure 8), and execution of the program outputs the values in the text boxes on the right of Figure 8. Inputting a value greater than zero will calculate the results in the output boxes. 


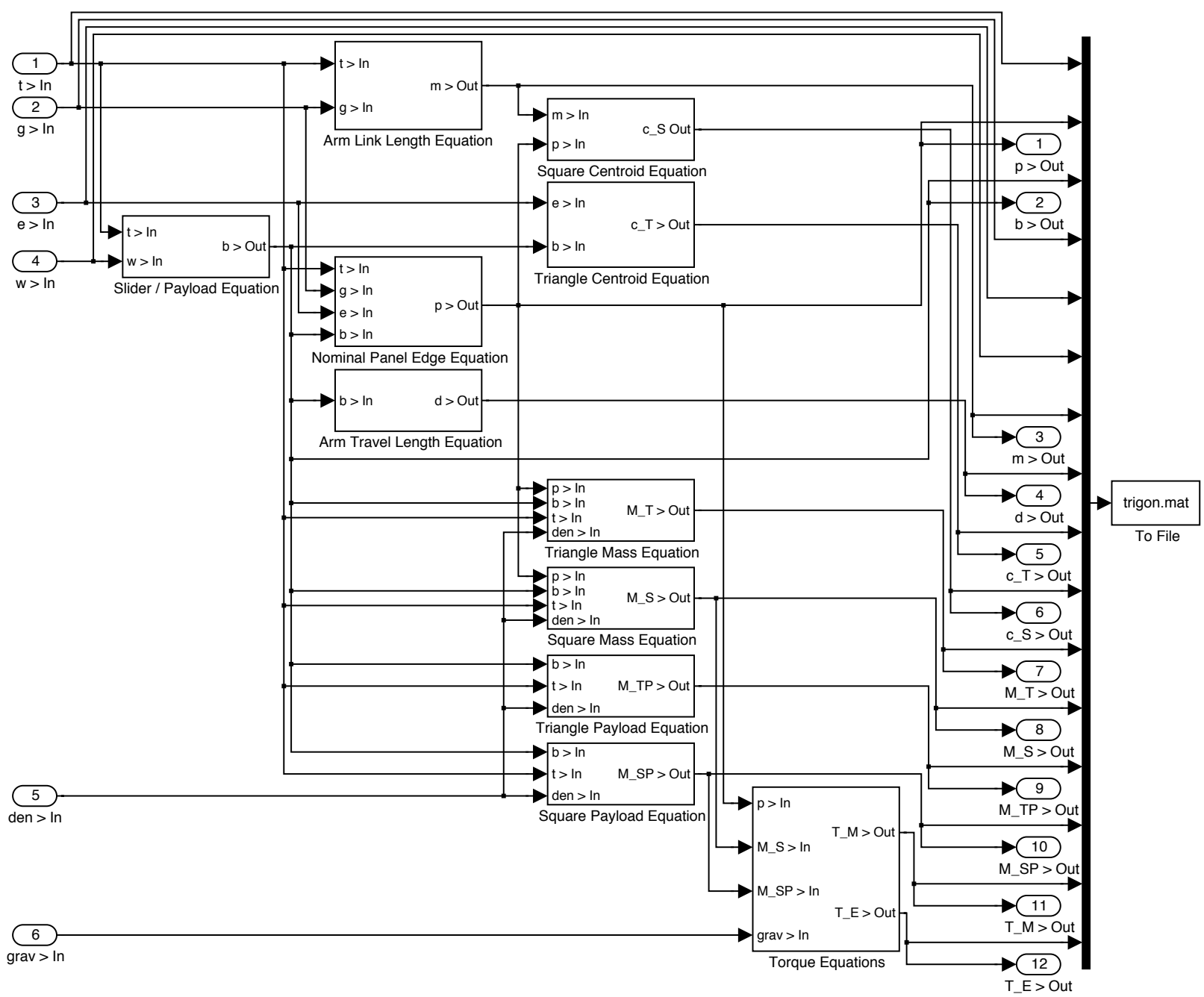

Figure 9: Panel sizing program geometry equations

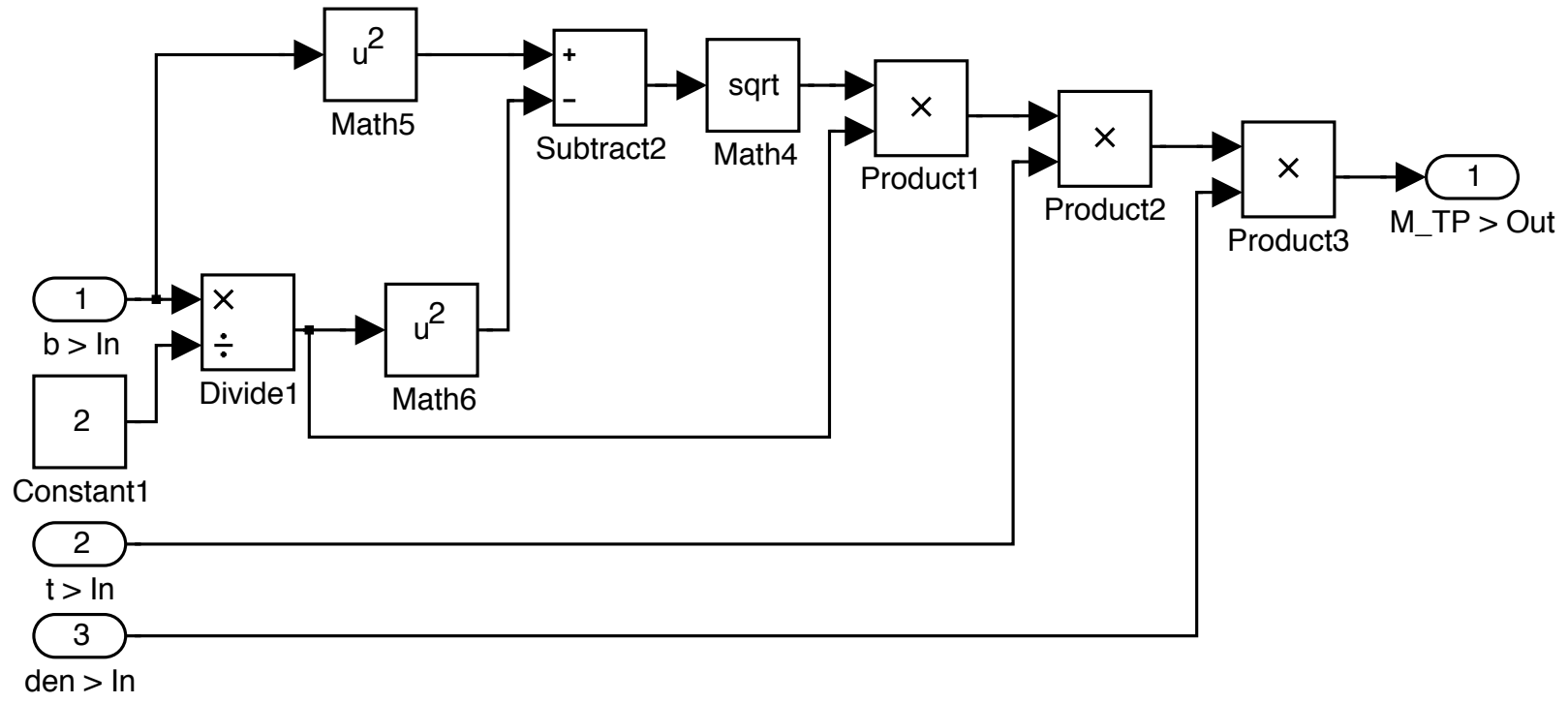

Figure 10: Panel sizing program mass calculation algorithm 
For the four input variables, if a number less than zero is inputted, the program will loop in an iterative manner and output the values to a spreadsheet file.

The mathematical model program consists of object-oriented coding that passes output values of one object to the input port of other objects, until all the equations have been visited. One example object is the equation for calculating the rest mass of one of the panels (Figure 10). The various equation objects are placed in a flow process that generates the output to either text boxes or file (Figure 9).

\section{Analysis}

In the course of the investigation, a partial spreadsheet was generated for panels ranging from $10 \mathrm{~cm}$ edge to $1.4 \mathrm{~m}$ edge dimension, in Earth gravity. A selected portion of the spreadsheet is shown in Table 1. The spreadsheet was generated by setting two of the three parameters of thickness, depth, or width to a set dimension, and incrementing the third dimension up to a pre-selected ceiling. The ceiling was arbitrarily set as $w: 2 t: 10 e$, or in other words, the value of $w$ may be up to twice the value of $t$, or 10 times the value of $e$. With the model in place, these ceilings may be adjusted later as new information is made available.

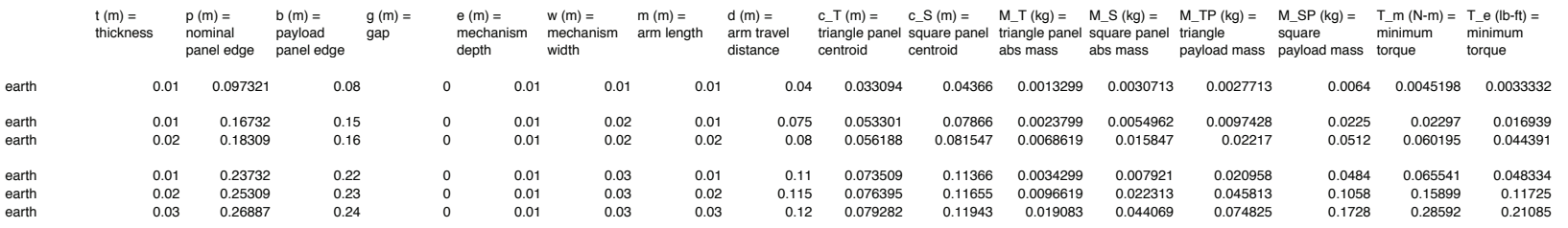

Table 1: Panel sizing spreadsheet (selection)

The graph in Figure 11 shows a comparison of panel overall dimension, panel rest mass, and minimum torque. The graph consists of broken curves due to the step up of parameters in each set. In other words, a panel thickness beginning at $0.01 \mathrm{~m}$ stepped up to $0.1 \mathrm{~m}$, for example, until the value reaches one of the ceilings proportional to the other two input values, then starts at $0.01 \mathrm{~m}$ again after stepping up the other value.

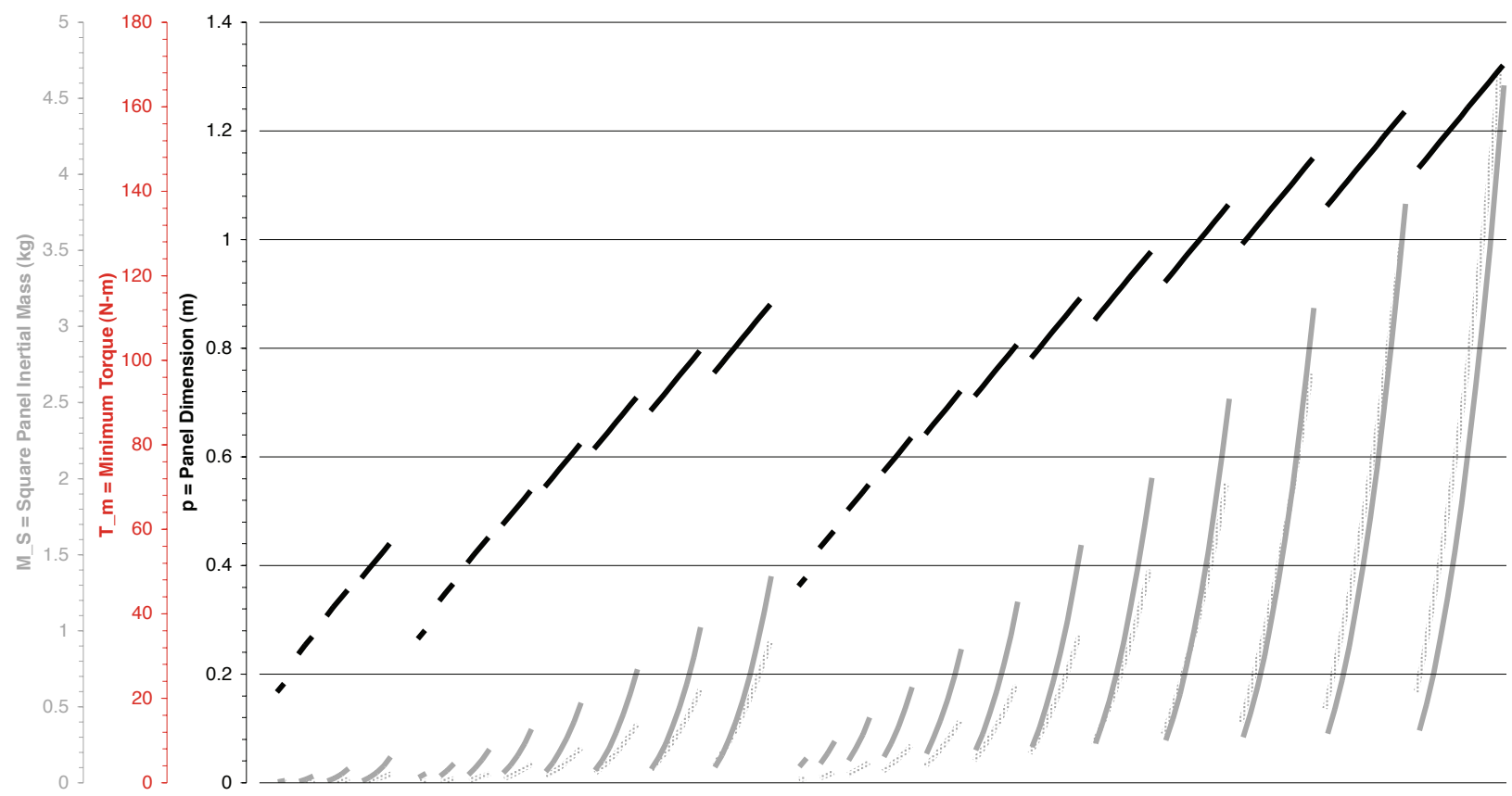

Figure 11: Comparison graph for panel size, mass, and connector arm torque

The spreadsheet and graph will be helpful in the further development of the Trigon technology by mapping out ranges where torque is kept to reasonable values, or ergonomic features are possible (such as accommodating hatches), etc. For example, torque ranges in the Figure 11 graph may be considered unreasonable, even with high- 
ratio reduction gearing systems, at values over $30 \mathrm{~N}-\mathrm{m}$ (however, this will depend on the size of the motor selected, and exotic solutions that can accommodate values outside the range may still be possible).

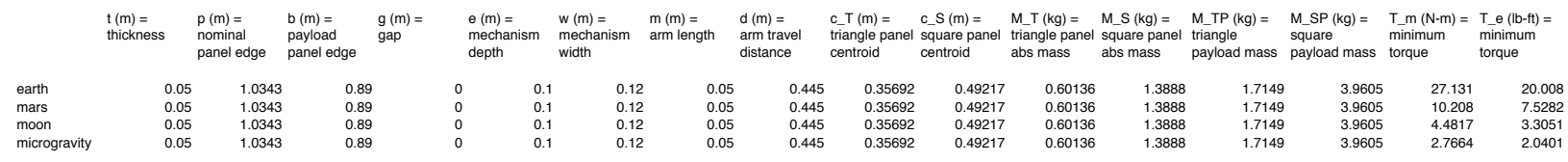

Table 2: Torque comparisons for variable gravity accelerations

Table 2 shows the comparison of torque values for four different gravities: Earth, Mars, Moon, and microgravity. Note that the mass values of each reference frame acceleration remains the same; this value would be multiplied by the gravity acceleration to determine equivalent forces in each of the environments.

\section{Conclusion}

A tool in the form of an object-oriented mathematical parametric model was devised for generating multivariate dimensional values for the purpose of determining optimum panel sizes for the Trigon panels. The tool will be used for future research, where rigorous output will be required for exploring a full range of options on motor selection, reduction gear mechanism design, and payload panel sizing. The importance of having a panel size optimization analysis tool lies clearly in the fact that most of the actual constraints for designing optimally sized panels are unknown at this time. This analysis therefore takes into account future unknowns and allows a selection of a variety of sizes for different needs.

\section{References}

M.M. Cohen; K.J. Kennedy (1997). Habitats \& Surface Construction Technology \& Development Roadmap. Proceedings of Exploration Technology Team Meeting, Executive Summary. 10 July, 1997. NASA Johnson Space Center.

A.S. Howe (2002). The Ultimate Construction Toy: Applying Kit-of-Parts Theory to Habitat and Vehicle Design (AIAA 2002-6116). 1st Space Architecture Symposium (SAS 2002), Houston, Texas, USA, 10-11 October 2002. Reston, Virginia, USA: American Institute of Aeronautics and Astronautics.

A.S. Howe (2005). Cassette Factories and Robotic Bricks: a Roadmap for Establishing Deep Space Infrastructures (SAE 2005-01-2911). Proceedings of the 35th International Conference on Environmental Systems (ICES2005), 11-14 July 2005, Rome, Italy. 400 Commonwealth Drive, Warrendale, PA: Society of Automotive Engineers.

A.S. Howe; I. Gibson (2006a). MOBITAT2: A Mobile Habitat Based on the Trigon Construction System (AIAA 20067337). 2nd International Space Architecture Symposium. San Jose, California, USA, 19-21 September 2006. Reston, Virginia, USA: American Institute of Aeronautics and Astronautics.

A.S. Howe; I. Gibson (2006b). Trigon Robotic Pairs (AIAA 2006-7407). AIAA Space 2006 Conference \& Exhibition. San Jose, California, USA, 19-21 September 2006. Reston, Virginia, USA: American Institute of Aeronautics and Astronautics.

K.J. Kennedy (2002). The Vernacular of Space Architecture (AIAA 2002-6102). 1st Space Architecture Symposium (SAS 2002), Houston, Texas, USA, 10-11 October 2002. Reston, Virginia, USA: American Institute of Aeronautics and Astronautics.

Y. Lai; A.S. Howe (2003). A Kit-of-parts Approach to Pressure Vessels for Planetary Surface Construction (AIAA 20036281). AIAA Space 2003 Conference \& Exposition, Long Beach, California, USA, 23-25 September 2003. Reston, Virginia, USA: American Institute of Aeronautics and Astronautics.

W. Yip; A.S. Howe (2003). Deployable Secondary Support Structures for Planetary Construction (AIAA 2003-6282). AIAA Space 2003 Conference \& Exposition, Long Beach, California, USA, 23-25 September 2003. Reston, Virginia, USA: American Institute of Aeronautics and Astronautics.

\section{Nomenclature}

AIAA $=$ American Institute of Aeronautics and Astronautics

$I S R U=$ In-situ Resource Utilization

$N A S A=$ National Aeronautics and Space Administration

Trigon $=$ Transformable Robotic Infrastructure-Generating Object Network system 